\title{
Robust Asymptotic Stabilization of Hybrid Systems using Control Lyapunov Functions
}

\author{
Ricardo G. Sanfelice \\ Computer Engineering Department \\ University of California \\ Santa Cruz, California, USA \\ ricardo@ucsc.edu
}

\begin{abstract}
We propose tools for the study of robust stabilizability and the design of robustly stabilizing feedback laws for a wide class of hybrid systems given in terms of hybrid inclusions with inputs and disturbances. We introduce notions of robust uniform global stabilizability and stabilization that capture the case when disturbances can be fully rejected, practically rejected, and when they induce a residual set that can be stabilized. Robust control Lyapunov functions are employed to determine when stabilizing static state-feedback laws are available and also to synthesize robustly stabilizing feedback laws with minimum pointwise norm. Sufficient conditions on the data of the hybrid system as well as on the control Lyapunov function are proposed for the said properties to hold. An example illustrates the results throughout the paper.
\end{abstract}

\section{CCS Concepts}

-Theory of computation $\rightarrow$ Timed and hybrid models; •Computing methodologies $\rightarrow$ Control methods; $\bullet$ Hardware $\rightarrow$ Process variations;

\section{Keywords}

Hybrid systems; Robust stability; Control Lyapunov functions

\section{INTRODUCTION}

Recent advances in the theory of hybrid dynamical systems have provided powerful tools for the study of robustness of asymptotic stability. One of the main results in [7], which is for hybrid systems modeled as hybrid inclusions,

\footnotetext{
* Research partially supported by the National Science Foundation under CAREER Grant no. ECCS-1450484 and Grant no. CNS-1544396, and by the Air Force Office of Scientific Research under YIP Grant no. FA9550-12-1-0366 and Grant no. FA9550-16-1-0015.
}

Permission to make digital or hard copies of all or part of this work for personal or classroom use is granted without fee provided that copies are not made or distributed for profit or commercial advantage and that copies bear this notice and the full citation on the first page. Copyrights for components of this work owned by others than ACM must be honored. Abstracting with credit is permitted. To copy otherwise, or republish, to post on servers or to redistribute to lists, requires prior specific permission and/or a fee. Request permissions from permissions@acm.org.

HSCC'16, April 12-14, 2016, Vienna, Austria

(C) 2016 ACM. ISBN 978-1-4503-3955-1/16/04 .. \$15.00 DOI: http://dx.doi.org/10.1145/2883817.2883848 is that asymptotic stability of a compact set is nominally robust when the objects defining the hybrid system satisfy mild regularity properties - by nominal robustness we mean that the stability property is be preserved semiglobally and practically for small enough perturbations. The importance of this result for control design is significant, as it highlights structural properties that the interconnection between the plant and the controller (both potentially hybrid) should satisfy so that, after a perturbation-free design, the behavior of the closed-loop system does not change much when small perturbations are present (even when those perturbations may affect the times at which flows and jumps occur). The case of large disturbances in hybrid systems was studied in 1 using the notion of input-to-state stability (ISS). While the results therein involving ISS Lyapunov functions can certainly be used for design, constructive design tools that guarantee robustness of asymptotic stability to large disturbances are not yet available.

Control Lyapunov functions have been shown to be very useful in constructively designing feedback control algorithms 14, 3, 13, 6]. In particular, in 6], tools for the design of robustly stabilizing feedback controllers are proposed for continuous-time systems for which a robust control Lyapunov function exists. A salient feature of using robust control Lyapunov functions is that, even under the presence of large disturbances, an asymptotic stability of a set, typically defined by a residual neighborhood around the desired equilibrium, can be guaranteed. Recently, the concept of control Lyapunov function was extended to different classes of hybrid systems without disturbances, see [11 for results for hybrid inclusions and [4] for results for discrete-time systems with continuous and discrete states.

Motivated by the constructive design tools for robust stability in [6], in this paper, we propose tools for the study of robust stabilizability and the design of robustly stabilizing feedback laws that employ control Lyapunov functions for hybrid systems with disturbances. For a wide class of hybrid systems given in terms of hybrid inclusions with inputs and disturbances, we introduce notions of robust uniform global stabilizability and stabilization that capture the case when disturbances can be fully rejected, practically rejected, and when they induce a residual set that can be stabilized. Building from results in [1], we propose conditions guaranteeing the existence of a continuous robust stabilizing static state-feedback law. We show that, under further conditions, continuous state-feedback laws with minimum pointwise norm can be constructed. 
The remainder of this paper is organized as follows. In Section 2 we introduce the hybrid system model and related notions. The notions of robust stability, stabilizability, and control Lyapunov functions are introduced in Section 3 . Conditions guaranteeing the existence of stabilizing feedback laws are given in Section 4 while the constructive design tools are in Section 5. Due to space constraints, the proof of the results are not included but will be published elsewhere.

Notation: $\mathbb{R}^{n}$ denotes $n$-dimensional Euclidean space. $\mathbb{R}$ denotes the real numbers. $\mathbb{R}_{\geq 0}$ denotes the nonnegative real numbers, i.e., $\mathbb{R}_{\geq 0}=[0, \infty) . \mathbb{N}$ denotes the natural numbers including 0, i.e., $\mathbb{N}=\{0,1, \ldots\}$. $\mathbb{B}$ denotes the closed unit ball in a Euclidean space. Given a set $K, \bar{K}$ denotes its closure. Given a set $S, \partial S$ denotes its boundary. Given $x \in \mathbb{R}^{n},|x|$ denotes the Euclidean vector norm. Given a closed set $K \subset \mathbb{R}^{n}$ and $x \in \mathbb{R}^{n},|x|_{K}:=\inf _{y \in K}|x-y|$. Given vectors $x$ and $y,\langle x, y\rangle$ denotes their inner product and, at times, we write $\left[x^{\top} y^{\top}\right]^{\top}$ simply as $(x, y)$. A function $\rho: \mathbb{R}^{n} \rightarrow \mathbb{R}_{\geq 0}$ is positive definite with respect to a set $S$ if $\rho(x)=0$ for each $x \in S$ and $\rho(x)>0$ for each $x \in \mathbb{R}^{n} \backslash S$. A function $\alpha: \mathbb{R}_{\geq 0} \rightarrow \mathbb{R}_{\geq 0}$ is said to belong to class- $\mathcal{K}$ if it is continuous, zero at zero, and strictly increasing. A function $\alpha: \mathbb{R}_{\geq 0} \rightarrow \mathbb{R}_{\geq 0}$ is said to belong to class- $\mathcal{K}_{\infty}$ if it is an unbounded class- $\mathcal{K}$ function. A function $\beta: \mathbb{R}_{\geq 0} \times$ $\mathbb{R}_{\geq 0} \rightarrow \mathbb{R}_{\geq 0}$ is a class- $\mathcal{K} \mathcal{L}$ function, also written $\beta \in \mathcal{K} \mathcal{L}$, if it is nondecreasing in its first argument, nonincreasing in its second argument, $\lim _{r \rightarrow 0^{+}} \beta(r, s)=0$ for each $s \in$ $\mathbb{R}_{\geq 0}$, and $\lim _{s \rightarrow \infty} \beta(r, s)=0$ for each $r \in \mathbb{R}_{\geq 0}$. Given a locally Lipschitz function $V: \mathbb{R}^{n} \rightarrow \mathbb{R}_{\geq 0}, V^{\circ}(x ; \xi)$ denotes the Clarke generalized derivative of $V$ at $x$ in the direction of $\xi$; see 2]. Given a map $f$, its graph is denoted by $\operatorname{gph}(f)$. Given a set $S \subset \mathbb{R}_{\geq 0} \times \mathbb{N}, \sup _{t} S:=\sup \{t:(t, j) \in S\}$ and $\sup _{j} S:=\sup \{j:(t, j) \in S\}$.

\section{HYBRID SYSTEMS WITH INPUTS AND DISTURBANCES}

A hybrid system $\mathcal{H}_{u, w}$ with state $x$, control input $u=$ $\left(u_{c}, u_{d}\right)$, and disturbance input $w=\left(w_{c}, w_{d}\right)$ is given by

$$
\mathcal{H}_{u, w}\left\{\begin{array}{llll}
\dot{x} & \in & F\left(x, u_{c}, w_{c}\right) & \left(x, u_{c}, w_{c}\right) \in C \\
x^{+} \in & G\left(x, u_{d}, w_{d}\right) & \left(x, u_{d}, w_{d}\right) \in D
\end{array}\right.
$$

The space for the state $x$ is $\mathbb{R}^{n}$, the space for the input $u=$ $\left(u_{c}, u_{d}\right)$ is $\mathcal{U}=\mathcal{U}_{c} \times \mathcal{U}_{d}$, where $\mathcal{U}_{c} \subset \mathbb{R}^{m_{c}}$ and $\mathcal{U}_{d} \subset \mathbb{R}^{m_{d}}$, and the space for the disturbance $w=\left(w_{c}, w_{d}\right)$ is $\mathcal{W}=\mathcal{W}_{c} \times \mathcal{W}_{d}$, where $\mathcal{W}_{c} \subset \mathbb{R}^{d_{c}}$ and $\mathcal{W}_{d} \subset \mathbb{R}^{d_{d}}$. The data defining $\mathcal{H}_{u, w}$ is as follows:

- The set $C \subset \mathbb{R}^{n} \times \mathcal{U}_{c} \times \mathcal{W}_{c}$ is the flow set;

- The set-valued map $F: \mathbb{R}^{n} \times \mathbb{R}^{m_{c}} \times \mathbb{R}^{d_{c}} \rightrightarrows \mathbb{R}^{n}$ is the flow map;

- The set $D \subset \mathbb{R}^{n} \times \mathcal{U}_{d} \times \mathcal{W}_{d}$ is the jump set;

- The set-valued map $G: \mathbb{R}^{n} \times \mathbb{R}^{m_{d}} \times \mathbb{R}^{d_{d}} \rightrightarrows \mathbb{R}^{n}$ is the jump map.

The sets $C$ and $D$ in the definition of $\mathcal{H}_{u, w}$ define conditions that $x, u$, and $w$ should satisfy for flows or jumps to occur. Throughout this paper, we assume that these sets impose conditions on $u$ that only depend on $x$ and conditions on $w$ that only depend on $x$.

The state $x$ of the hybrid system can include multiple logic variables, timers, memory states as well as physical (continuous) states, e.g., $x=(q, \tau, \xi)$ is a state vector with a state component given by a logic variable $q$ taking values from a discrete set $\mathcal{Q}$, a state component given by a timer $\tau$ taking values from the interval $\left[0, \tau^{*}\right]$, where $\tau^{*}>0$ is the maximum allowed value for the timer, and with a state component $\xi \in \mathbb{R}^{n_{p}}$ representing the continuously varying state - note that in such a case, $\mathcal{Q} \times\left[0, \tau^{*}\right] \times \mathbb{R}^{n_{p}}$ can be embedded in $\mathbb{R}^{n}$ for $n=1+1+n_{p}$.

Given a set $K \subset \mathbb{R}^{n} \times \mathcal{U}_{\star} \times \mathcal{W}_{\star}$ with $\star$ being either $c$ or $d$, $\mathcal{U}_{\star} \subset \mathbb{R}^{m_{\star}}, \mathcal{W}_{\star} \subset \mathbb{R}^{d_{\star}}, V: \mathbb{R}^{n} \rightarrow \mathbb{R}_{\geq 0}$, and $r \geq 0$, we define

- $\mathcal{I}(r):=\left\{x \in \mathbb{R}^{n}: V(x) \geq r\right\}$

- The projector onto the state space

$$
\Pi_{\star}(K):=\left\{x: \exists\left(u_{\star}, w_{\star}\right) \text { s.t. }\left(x, u_{\star}, w_{\star}\right) \in K\right\}
$$

- The projector onto the state and input space

$$
\begin{aligned}
\Delta_{\star}(r, K):= & \left\{\left(x, u_{\star}\right): \exists w_{\star}\right. \text { s.t. } \\
& \left.\left(x, u_{\star}, w_{\star}\right) \in K \cap\left(\mathcal{I}(r) \times \mathbb{R}^{m_{\star}} \times \mathbb{R}^{d_{\star}}\right)\right\}
\end{aligned}
$$

- The projector onto the input and disturbance space

$$
\widetilde{\Psi}_{\star}^{u}(x, K):=\left\{u_{\star}^{\prime}: \exists w_{\star}^{\prime} \text { s.t. }\left(x, u_{\star}^{\prime}, w_{\star}^{\prime}\right) \in K\right\}
$$

and

$$
\widetilde{\Psi}_{\star}^{w}(x, K):=\left\{w_{\star}^{\prime}: \exists u_{\star}^{\prime} \text { s.t. }\left(x, u_{\star}^{\prime}, w_{\star}^{\prime}\right) \in K\right\}
$$

for each $x \in \mathbb{R}^{n}$, respectively;

- The projector onto the flow input, flow disturbance, jump input, and jump disturbance space

$$
\begin{aligned}
& \Psi_{c}^{u}(x):=\widetilde{\Psi}_{c}^{u}(x, C), \quad \Psi_{c}^{w}(x):=\widetilde{\Psi}_{c}^{w}(x, C) \\
& \Psi_{d}^{u}(x):=\widetilde{\Psi}_{d}^{u}(x, D), \quad \Psi_{d}^{w}(x):=\widetilde{\Psi}_{d}^{w}(x, D)
\end{aligned}
$$

for each $x \in \mathbb{R}^{n}$, respectively.

That is, given a set $K, \Pi_{\star}(K)$ denotes the "projection" of $K$ onto $\mathbb{R}^{n}, \Delta_{\star}(r, K)$ denotes the "projection" of $K$ onto $\left(\mathbb{R}^{n} \cap \mathcal{I}(r)\right) \times \mathbb{R}^{m_{\star}}$, while, given $x, \widetilde{\Psi}_{\star}^{u}(x, K)$ denotes the set of values $u_{\star}$ such that $\left(x, u_{\star}, w_{\star}\right) \in K$; similarly for $\widetilde{\Psi}_{\star}^{w}(x, K)$.

Solutions to hybrid systems $\mathcal{H}_{u, w}$ are given in terms of hybrid arcs, hybrid disturbances, and hybrid inputs on hybrid time domains. A set $\mathcal{E} \subset \mathbb{R}_{\geq 0} \times \mathbb{N}$ is a compact hybrid time domain if

$$
\mathcal{E}=\bigcup_{j=0}^{J-1}\left(\left[t_{j}, t_{j+1}\right], j\right)
$$

for some finite sequence of times $0=t_{0} \leq t_{1} \leq t_{2} \leq \ldots \leq t_{J}$. It is a hybrid time domain if for all $(T, J) \in \mathcal{E}$,

$$
\mathcal{E} \cap([0, T] \times\{0,1, \ldots, J\})
$$

is a compact hybrid time domain $\mathrm{A}$ hybrid $\operatorname{arc} \phi$ is a function on a hybrid time domain that, for each $j \in \mathbb{N}$, ${ }^{1}$ This property is to hold at each $(T, J) \in \mathcal{E}$, but $\mathcal{E}$ can be
unbounded. 
$t \mapsto \phi(t, j)$ is absolutely continuous on the interval

$$
\{t:(t, j) \in \operatorname{dom} \phi\}
$$

where dom $\phi$ denotes the hybrid time domain of $\phi$.

Hybrid disturbances $w$ are functions of hybrid time that will be generated by some hybrid exosystem $\mathcal{H}_{e}$ of the form

$$
\mathcal{H}_{e}\left\{\begin{array}{llll}
\dot{w} & \in & F_{e}(w) & w \in C_{e} \\
w^{+} & \in & G_{e}(w) & w \in D_{e}
\end{array}\right.
$$

with state (and output) $w=\left(w_{c}, w_{d}\right) \in \mathcal{W}$. A disturbance generated by a hybrid exosystem of the form (2) that, for given state trajectory and input, satisfies the dynamics of the hybrid system $\mathcal{H}_{u, w}$ is said to be admissible. For instance, the hybrid exosystem with data

$$
C_{e}=D_{e}=\mathcal{W}_{c} \times \mathcal{W}_{d}, \quad G_{e} \equiv \mathcal{W}_{c} \times \mathcal{W}_{d}, \quad F_{e} \equiv c \mathbb{B}
$$

where $c \geq 0$ is a constant, generates disturbances that remain in $\mathcal{W}$ and that are Lipschitz continuous during flows (with Lipschitz constant $c$ ), but not necessarily differentiable; see [9] for constructions of hybrid exosystems generating square and triangular signals.

Similarly, control inputs $u$ are functions of hybrid time, i.e., $u$ : $\operatorname{dom} u \rightarrow \mathcal{U}$ with $\operatorname{dom} u$ being a hybrid time domain, with the property that, for each $j, t \mapsto u(t, j)$ is Lebesgue measurable and locally essentially bounded on the interval $\{t:(t, j) \in \operatorname{dom} u\}$. A control input satisfying these properties and, for given state trajectory and disturbance, satisfies the dynamics of the hybrid system $\mathcal{H}_{u, w}$ is said to be admissible.

A solution to the hybrid system $\mathcal{H}_{u, w}$ in (1) is given by $(\phi, u, w), u=\left(u_{c}, u_{d}\right), w=\left(w_{c}, w_{d}\right)$, with $\operatorname{dom} \phi=\operatorname{dom} u=$ $\operatorname{dom} w(=\operatorname{dom}(\phi, u, w))$ and satisfying the dynamics of $\mathcal{H}_{u, w}$, where $\phi$ is a hybrid arc, $u$ is a hybrid input, and $w$ is a hybrid disturbance. A solution $(\phi, u, w)$ to $\mathcal{H}_{u, w}$ is said to be complete if $\operatorname{dom}(\phi, u, w)$ is unbounded, and is said to be maximal if there does not exist another pair $(\phi, u, w)^{\prime}$ such that $(\phi, u, w)$ is a truncation of $(\phi, u, w)^{\prime}$ to some proper subset of $\operatorname{dom}(\phi, u, w)^{\prime}$. For more details about solutions to hybrid systems with inputs, see [1].

Next, we illustrate the modeling framework in a system that will be revisited throughout the paper. Being of second order, with jumps in both of its state variables, and exhibiting Zeno behavior for specific choices of its inputs, the system is rich enough, yet not overly complex, for the purposes of illustrating our ideas and results.

EXAMPLE 2.1. (controlled pendulum with impacts) Consider a point-mass pendulum impacting on a controlled slanted surface. Denote the pendulum's angle (with respect to the vertical) by $x_{1}$, where $x_{1}>0$ corresponds to a displacement to the right of the vertical and $x_{1}<0$ to a displacement to the left of the vertical. The pendulum's velocity (positive when the pendulum rotates in the counterclockwise direction) is denoted by $x_{2}$. When $x_{1} \geq \mu$ with $\mu$ denoting the angle of the surface, its continuous evolution is given by

$$
\begin{aligned}
& \dot{x}_{1}=x_{2} \\
& \dot{x}_{2}=-a \sin x_{1}-\left(b+w_{c, 2}\right) x_{2}+\tau+w_{c, 1}
\end{aligned}
$$

where $a>0, b \geq 0$ capture the system constants (e.g., gravity, mass, length, and friction) and $\tau$ corresponds to torque actuation at the pendulum's end. For simplicity, we assume that $x_{1} \in\left[-\frac{\pi}{2}, \frac{\pi}{2}\right]$ and $\mu \in\left[-\frac{\pi}{2}, 0\right]$. The disturbance $w_{c, 1}$ represents actuator noise and unmodeled dynamics, while $w_{c, 2}$ represents uncertainty in the damping constant $b$. Impacts between the pendulum and the surface occur when

$$
x_{1} \leq \mu, \quad x_{2} \leq 0 .
$$

At such events, the jump map takes the form

$$
\begin{aligned}
& x_{1}^{+}=x_{1}+\widetilde{\rho}(\mu) x_{1} \\
& x_{2}^{+}=-\left(e(\mu)+w_{d}\right) x_{2}
\end{aligned}
$$

where the functions

$$
\widetilde{\rho}:[-\pi / 2,0] \rightarrow(-1,0)
$$

and

$$
e:[-\pi / 2,0] \rightarrow\left[e_{0}, e_{1}\right]
$$

$0<e_{0}<e_{1}<1$, are linear in $\mu$ and capture the effect of pendulum compression and restitution at impacts, respectively, as a function of $\mu$. For simplicity, the function $\widetilde{\rho}$ is used to capture (much more complex) rapid displacements of the pendulum at collisions by guaranteeing that $x_{1}+\widetilde{\rho}(\mu) x_{1}>x_{1}$ at jumps - in this way, after impacts away from $x_{1}=0$, the pendulum is pushed away from the contact condition. The restitution coefficient function e models the effect of gravity on energy dissipation at impacts via the angle $\mu$ : when the surface is placed as far to the left as possible $(\mu=-\pi / 2)$, e is given by the minimum value $e(-\pi / 2)=e_{0}$, while when the surface is at $\mu=0$, e takes the maximum value $e(0)=e_{1}$. The disturbance $w_{d}$ represents uncertainty in the restitution coefficient.

The model above can be captured by the hybrid system $\mathcal{H}_{u, w}$ given by

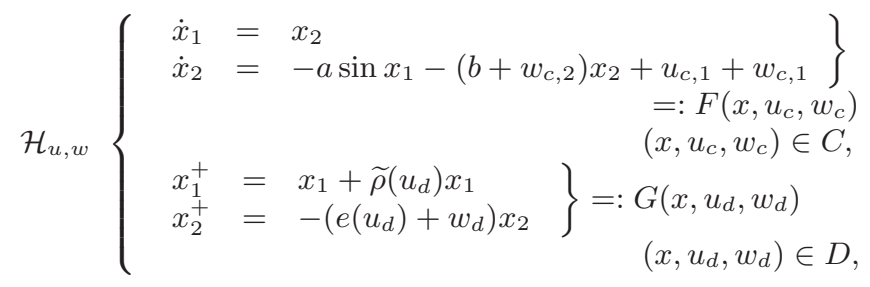

where $u_{c}=\left[\begin{array}{ll}u_{c, 1} & u_{c, 2}\end{array}\right]^{\top}=\left[\begin{array}{ll}\tau & \mu\end{array}\right]^{\top} \in \mathbb{R} \times\left[-\frac{\pi}{2}, 0\right]=: \mathcal{U}_{c}, u_{d}=$ $\mu \in\left[-\frac{\pi}{2}, 0\right] \stackrel{=}{=} \mathcal{U}_{d}, w_{c}=\left(w_{c, 1}, w_{c, 2}\right) \in \mathcal{W}_{c}:=\left[0, \bar{w}_{1}\right] \times\left[0, \bar{w}_{2}\right]$ with $\bar{w}_{1}, \bar{w}_{2} \in \mathbb{R}_{\geq 0}, w_{d} \in \mathcal{W}_{d}:=\left[0, e_{1}-e_{0}\right]$,

$$
\begin{gathered}
C:=\left\{\left(x, u_{c}, w_{c}\right) \in\left[-\frac{\pi}{2}, \frac{\pi}{2}\right] \times \mathbb{R} \times \mathcal{U}_{c} \times \mathcal{W}_{c}: x_{1} \geq u_{c, 2}\right\}, \\
D:=\left\{\left(x, u_{d}, w_{d}\right) \in\left[-\frac{\pi}{2}, \frac{\pi}{2}\right] \times \mathbb{R} \times \mathcal{U}_{d} \times \mathcal{W}_{d}:\right. \\
\left.x_{1} \leq u_{d}, x_{2} \leq 0\right\}
\end{gathered}
$$

Note that the definitions of $C$ and $D$ impose state constraints on the inputs that only depend on the state $x$.

The following mild conditions on the data of $\mathcal{H}_{u, w}$ will be imposed in some of our results.

Definition 2.2. (hybrid basic conditions) A hybrid system $\mathcal{H}_{u, w}$ is said to satisfy the hybrid basic conditions if its data satisfies

(A1) $C$ and $D$ are closed subsets of $\mathbb{R}^{n} \times \mathcal{U}_{c} \times \mathcal{W}_{c}$ and $\mathbb{R}^{n} \times \mathcal{U}_{d} \times \mathcal{W}_{d}$, respectively; 
(A2) $F: \mathbb{R}^{n} \times \mathbb{R}^{m_{c}} \times \mathbb{R}^{d_{c}} \rightrightarrows \mathbb{R}^{n}$ is outer semicontinuous relative to $C$ and locally bounded ${ }^{2}$, and for all $\left(x, u_{c}, w_{c}\right) \in C, F\left(x, u_{c}, w_{c}\right)$ is nonempty and convex;

(A3) $G: \mathbb{R}^{n} \times \mathbb{R}^{m_{d}} \times \mathbb{R}^{d_{d}} \rightrightarrows \mathbb{R}^{n}$ is outer semicontinuous relative to $D$ and locally bounded, and for all $\left(x, u_{d}, w_{d}\right) \in D, G\left(x, u_{d}, w_{d}\right)$ is nonempty.

When $F$ is single valued, (A2) reduces to $F$ being continuous. Similarly, when $G$ is single valued, (A3) reduces to $G$ being continuous.

In the sections to follow, we will design state-feedback laws to control the hybrid system $\mathcal{H}_{u, w}$. The resulting closed-loop system under the effect of the control pair $\left(\kappa_{c}, \kappa_{d}\right)$ is given by

$\mathcal{H}_{c l} \begin{cases}\dot{x} \in F_{c l}\left(x, w_{c}\right):=F\left(x, \kappa_{c}(x), w_{c}\right) & \left(x, w_{c}\right) \in C_{c l} \\ x^{+} \in G_{c l}\left(x, w_{d}\right):=G\left(x, \kappa_{d}(x), w_{d}\right) & \left(x, w_{d}\right) \in D_{c l}\end{cases}$

with

$$
C_{c l}:=\left\{\left(x, w_{c}\right) \in \mathbb{R}^{n} \times \mathcal{W}_{c}:\left(x, \kappa_{c}(x), w_{c}\right) \in C\right\}
$$

and

$$
D_{c l}:=\left\{\left(x, w_{d}\right) \in \mathbb{R}^{n} \times \mathcal{W}_{d}:\left(x, \kappa_{d}(x), w_{d}\right) \in D\right\} .
$$

Note that when the components of $u_{c}$ and $u_{d}$ correspond to the same physical input, like $\mu$ in Example 2.1, such components of the feedback law pair $\left(\kappa_{c}, \kappa_{d}\right)$ have to be identical - see the revisited version of Example 2.1 in Example 5.4.

REMARK 2.3. When $\mathcal{H}_{u, w}$ satisfies the hybrid basic conditions and the state-feedback pair $\left(\kappa_{c}, \kappa_{d}\right)$ is continuous, the hybrid closed-loop system $\mathcal{H}_{c l}$ satisfies the hybrid basic conditions. An important consequence of $\mathcal{H}_{c l}$ satisfying the hybrid basic conditions is that asymptotic stability of a compact set for $\mathcal{H}_{u, w}$ (with $\left.w \equiv 0\right)$ is automatically nominally robust, in the sense that the asymptotic stability property is preserved (semiglobally and practically) under the presence of small enough perturbations.

\section{ROBUST STABILITY, STABILIZABILITY, AND CONTROL LYAPUNOV FUNCTIONS}

This section introduces the stability, stabilizability, and control Lyapunov function notions for $\mathcal{H}_{u, w}$ employed throughout the paper. Nominal versions of these notions can be found in [7] and [1].

First, we introduce a stability property of closed sets capturing robustness with respect to all admissible disturbances $w$. For simplicity, we write the global version, but, though more involved, a local version can certainly be formulated.

\footnotetext{
${ }^{2}$ A set-valued map $S: \mathbb{R}^{n} \rightrightarrows \mathbb{R}^{m}$ is outer semicontinuous at $x \in \mathbb{R}^{n}$ if for each sequence $\left\{x_{i}\right\}_{i=1}^{\infty}$ converging to a point $x \in \mathbb{R}^{n}$ and each sequence $y_{i} \in S\left(x_{i}\right)$ converging to a point $y$, it holds that $y \in S(x)$; see [10. Definition 5.4]. Given a set $X \subset \mathbb{R}^{n}$, it is outer semicontinuous relative to $X$ if the setvalued mapping from $\mathbb{R}^{n}$ to $\mathbb{R}^{m}$ defined by $S(x)$ for $x \in X$ and $\emptyset$ for $x \notin X$ is outer semicontinuous at each $x \in X$. It is locally bounded if for each compact set $K \subset \mathbb{R}^{n}$ there exists a compact set $K^{\prime} \subset \mathbb{R}^{n}$ such that $S(K):=\cup_{x \in K} S(x) \subset K^{\prime}$.
}

DEFINITION 3.1. (w-robust uniform global asymptotic stability) Given a control $u$, and closed sets $\mathcal{A}$ and $\widetilde{\mathcal{A}}$ subsets of $\mathbb{R}^{n}$, the set $\widetilde{\mathcal{A}}$ is said to be $w$-robustly uniformly globally asymptotically stable relative to $\mathcal{A}$ for the hybrid system $\mathcal{H}_{u, w}$ if

$$
\mathcal{A} \subset \widetilde{\mathcal{A}}
$$

and there exists $\beta \in \mathcal{K} \mathcal{L}$ such that, for each admissible disturbance $w$, every solution $\phi$ to $\mathcal{H}_{u, w}$ using the given control $u$ satisfies

$$
|\phi(t, j)|_{\widetilde{\mathcal{A}}} \leq \beta\left(|\phi(0,0)|_{\widetilde{\mathcal{A}}}, t+j\right) \quad \forall(t, j) \in \operatorname{dom} \phi
$$

REMARK 3.2. When the property in Definition 3.1 holds for $\widetilde{\mathcal{A}}=\mathcal{A}$, in which case we will drop "relative to $\mathcal{A}$," the notion resembles [7, Definition 3.6] with the addition that the property holds for every possible admissible disturbance. When $\mathcal{A} \neq \widetilde{\mathcal{A}}$, the set $\widetilde{\mathcal{A}}$ is a residual set relative to $\mathcal{A}$, meaning that complete solutions would converge to $\widetilde{\mathcal{A}}$ but may not converge to $\mathcal{A}$. A particular such situation is when $\mathcal{A}$ is the origin and the set $\widetilde{\mathcal{A}}$ is a small neighborhood around it. Finally, note that the property in Definition 3.1 and the ones introduced below, may hold for a large enough residual (e.g., $\widetilde{\mathcal{A}}=\mathbb{R}^{n}$ ), though one is typically interested in having $\widetilde{\mathcal{A}}$ to be some small neighborhood of $\mathcal{A}$.

REMARK 3.3. The property in Definition 3.1 differs from input-to-state stability (ISS) with respect to $w$ as the $\mathcal{K} \mathcal{L}$ bound defining ISS involves the distance from the state trajectory to a set (like $\mathcal{A}$ ), rather than to a residual set (like $\widetilde{\mathcal{A}})$, and includes an additive offset that is a function of a norm of w; see [1] for a definition of ISS for hybrid systems as in (1). A key difference is that ISS guarantees attractivity of a neighborhood of a set (of size depending on a norm of the disturbance), while our w-robust notion guarantees an asymptotic stability of a residual set that is uniform over all admissible disturbances.

The existence of some control $u$, perhaps (hybrid) time dependent, stabilizing a point or a set is known as stabilizability. Next, we introduce this notion for the case of hybrid systems under disturbances.

DEFINITION 3.4. (robust stabilizability) Given a hybrid system $\mathcal{H}_{u, w}$, a closed set $\mathcal{A} \subset \mathbb{R}^{n}$ is said to be

1) $w$-robustly uniformly globally asymptotically stabilizable for $\mathcal{H}_{u, w}$ if there exists an admissible control $u$ such that the set $\mathcal{A}$ is w-robustly uniformly globally asymptotically stable for $\mathcal{H}_{u, w}$;

2) $w$-robustly practically uniformly globally asymptotically stabilizable for $\mathcal{H}_{u, w}$ if for every $\varepsilon>0$ there exist an admissible control $u$ and a closed set $\widetilde{\mathcal{A}}$ satisfying

$$
\mathcal{A} \subset \widetilde{\mathcal{A}} \subset \mathcal{A}+\varepsilon \mathbb{B}
$$

such that the set $\widetilde{\mathcal{A}}$ is w-robustly uniformly globally asymptotically stable for $\mathcal{H}_{u, w}$ relative to $\mathcal{A}$;

3) $w$-robustly uniformly globally asymptotically stabilizable with residual $\widetilde{\mathcal{A}}$ for $\mathcal{H}_{u, w}$ with $\widetilde{\mathcal{A}}$ closed, $\mathcal{A} \subsetneq \widetilde{\mathcal{A}}$, if there exists an admissible control $u$ such that the set $\widetilde{\mathcal{A}}$ is w-robustly uniformly globally asymptotically stable relative to $\mathcal{A}$ for $\mathcal{H}_{u, w}$. 
REMARK 3.5. The notion in item 1) in Definition 3.4 captures the situation when the effect of the disturbances can be overcome and the desired set $\mathcal{A}$ rendered asymptotically stable by some control $u$. For the hybrid system in Example 2.1. for which the desired set $\mathcal{A}$ is naturally the origin, this set being $w$-robustly uniformly globally asymptotically stabilizable requires the existence of a control that renders the origin uniformly globally asymptotically stable for any disturbance $\left(w_{c}, w_{d}\right)$; see Example 5.4. The practical notion in item 2) corresponds to the situation when the asymptotically stable residual set $\widetilde{\mathcal{A}}$ can be made arbitrarily close to the set $\mathcal{A}$ by some control u. Finally, item 3) captures the situation when only a residual set can be stabilized.

Methods for synthesis of feedback control laws that induce the properties introduced above will employ control Lyapunov functions. For the nominal case, a control Lyapunov function for a hybrid system is a function that, for each value of the state, there exist control input values that make the function decrease during flows and jumps [11, Definition 2.1]. Following the construction in 6] Definition 3.8] for continuous-time systems, we introduce the following robust control Lyapunov function notion for $\mathcal{H}_{u, w}$.

DEFINITION 3.6. (robust control Lyapunov function) Given a closed set $\mathcal{A} \subset \mathbb{R}^{n}$, sets $\mathcal{U}_{c} \subset \mathbb{R}^{m_{c}}$ and $\mathcal{U}_{d} \subset \mathbb{R}^{m_{d}}$, and sets $\mathcal{W}_{c} \subset \mathbb{R}^{d_{c}}$ and $\mathcal{W}_{d} \subset \mathbb{R}^{d_{d}}$, a continuous function $V: \mathbb{R}^{n} \rightarrow \mathbb{R}$ that is locally Lipschitz on an open set containing $\overline{\Pi_{c}(C)}$ is a robust control Lyapunov function (RCLF) with $\mathcal{U}$ controls for $\mathcal{H}_{u, w}$ if there exis ${ }^{3} r^{*} \geq 0, \alpha_{1}, \alpha_{2} \in \mathcal{K}_{\infty}$, and a positive definite function $\alpha_{3}$ such that

$$
\begin{array}{r}
\alpha_{1}\left(|x|_{\mathcal{A}}\right) \leq V(x) \leq \alpha_{2}\left(|x|_{\mathcal{A}}\right) \\
\forall x \in \Pi_{c}(C) \cup \Pi_{d}(D) \cup G(D), \quad(7) \\
\inf _{u_{c} \in \Psi_{c}^{u}(x)} \sup _{w_{c} \in \Psi_{c}^{w}(x)} \sup _{\xi \in F\left(x, u_{c}, w_{c}\right)} V^{\circ}(x ; \xi) \leq-\alpha_{3}\left(|x|_{\mathcal{A}}\right) \\
\forall x \in \Pi_{c}(C) \cap \mathcal{I}(r), r \geq r^{*},(8) \\
\inf _{u_{d} \in \Psi_{d}^{u}(x)} \sup _{w_{d} \in \Psi_{d}^{w}(x)} \sup _{\xi \in G\left(x, u_{d}, w_{d}\right)} V(\xi)-V(x) \leq-\alpha_{3}\left(|x|_{\mathcal{A}}\right) \\
\forall x \in \Pi_{d}(D) \cap \mathcal{I}(r), r \geq r^{*} .
\end{array}
$$

EXAMPLE 3.7. (controlled pendulum with impacts (revisited)) For the hybrid system in Example 2.1. let $\mathcal{A}=\{(0,0)\}$ and consider the candidate robust control Lyapunov function with $\mathcal{U}$ controls for $\mathcal{H}_{u, w}$ given by

$$
V(x)=x^{\top} P x, \quad P=\left[\begin{array}{ll}
2 & 1 \\
1 & 1
\end{array}\right] .
$$

Condition (7) holds trivially. During flows, we have that

$$
\begin{aligned}
& \left\langle\nabla V(x), F\left(x, u_{c}, w_{c}\right)\right\rangle=4 x_{1} x_{2}+2 x_{2}^{2} \\
& +2\left(-a \sin x_{1}-\left(b+w_{c, 2}\right) x_{2}+u_{c, 1}+w_{c, 1}\right)\left(x_{2}+x_{1}\right)
\end{aligned}
$$

for all $\left(x, u_{c}, w_{c}\right) \in C$. It follows that (8) is satisfied with $\alpha_{3}$ defined as $\alpha_{3}(s):=s^{2}$ for all $s \geq 0$. In fact, note that,

\footnotetext{
${ }^{3}$ When $\mathcal{H}_{u, w}$ has purely continuous dynamics, i.e., it does not exhibit jumps, then $r \geq r^{*}$ can be replaced by $r>r^{*}$. In fact, in such a case, when $r^{*}=0$ solutions cannot flow out of $\mathcal{A}$. However, when the system has jumps, if (9) only holds for each $r>r^{*}=0$, there could still be solutions that jump outside of $\mathcal{A}$.
}

for each $x \in \mathbb{R}^{2}$,

$$
\begin{gathered}
\Psi_{c}^{u}(x)= \begin{cases}\mathbb{R} \times\left[-\frac{\pi}{2}, \min \left\{x_{1}, 0\right\}\right] & \text { if } x_{1} \in\left[-\frac{\pi}{2}, \frac{\pi}{2}\right] \\
\emptyset & \text { if } x_{1} \notin\left[-\frac{\pi}{2}, \frac{\pi}{2}\right]\end{cases} \\
\Psi_{c}^{w}(x)= \begin{cases}\mathcal{W}_{c} & \text { if } x_{1} \in\left[-\frac{\pi}{2}, \frac{\pi}{2}\right] \\
\emptyset & \text { if } x_{1} \notin\left[-\frac{\pi}{2}, \frac{\pi}{2}\right]\end{cases}
\end{gathered}
$$

and that $\Pi_{c}(C)=\left[-\frac{\pi}{2}, \frac{\pi}{2}\right] \times \mathbb{R}$. Then

$$
\inf _{u_{c} \in \Psi_{c}^{u}(x)} \sup _{w_{c} \in \Psi_{c}^{w}(x)}\left\langle\nabla V(x), F\left(x, u_{c}, w_{c}\right)\right\rangle=-x^{\top} x
$$

for all $x \in \Pi_{c}(C)$ such that $x_{1}+x_{2}=0$, while when $x_{1}+x_{2} \neq$ 0 , we have

$$
\inf _{u_{c} \in \Psi_{c}^{u}(x)} \sup _{w_{c} \in \Psi_{c}^{w}(x)}\left\langle\nabla V(x), F\left(x, u_{c}, w_{c}\right)\right\rangle=-\infty .
$$

For each $x \in \mathbb{R}^{2}$, we have

$$
\begin{gathered}
\Psi_{d}^{u}(x)= \begin{cases}{\left[x_{1}, 0\right]} & \text { if } x_{1} \in\left[-\frac{\pi}{2}, 0\right], x_{2} \leq 0 \\
\emptyset & \text { otherwise }\end{cases} \\
\Psi_{d}^{w}(x)= \begin{cases}\mathcal{W}_{d} & \text { if } x_{1} \in\left[-\frac{\pi}{2}, 0\right], x_{2} \leq 0 \\
\emptyset & \text { otherwise, }\end{cases}
\end{gathered}
$$

and that $\Pi_{d}(D)=\left[-\frac{\pi}{2}, 0\right] \times(-\infty, 0]$. Then, at jumps, we have

$$
\inf _{u_{d} \in \Psi_{d}^{u}(x)} \sup _{w_{d} \in \Psi_{d}^{w}(x)} V\left(G\left(x, u_{d}, w_{d}\right)\right)-V(x) \leq-\lambda x^{\top} x
$$

for all $x \in \Pi_{d}(D)$, where

$$
\lambda:=\min _{\eta_{1} \in\left[-\frac{\pi}{2}, 0\right]}\left\{2\left(1-\left(1+\widetilde{\rho}\left(\eta_{1}\right)\right)^{2}\right), 1-\left(e\left(\eta_{1}\right)+e_{1}-e_{0}\right)^{2}\right\}
$$

which, by the properties of $\widetilde{\rho}$ and $e$, is positive. Then, condition (9) is satisfied with $\alpha_{3}$ defined as $\alpha_{3}(s):=\lambda s^{2}$ for all $s \geq 0$.

It follows that both (8) and (9) hold with this choice of $\alpha_{3}$.

\section{ROBUST STABILIZABILITY VIA STATIC STATE-FEEDBACK LAWS}

In this section, we provide conditions guaranteeing the existence of a robustly stabilizing control $u$ inducing some of the properties introduced in Section 3 Our interest is in control laws that are of (static) state-feedback type and continuous, which, as argued in Remark 2.3 when $\mathcal{H}_{u, w}$ satisfies the hybrid basic conditions, would lead to a closedloop system $\mathcal{H}_{c l}$ (without $u$ ) as in (4) satisfying the hybrid basic conditions.

Given the compact set $\mathcal{A}$ and a robust control Lyapunov function $V$ satisfying Definition 3.6 with positive definite function $\alpha_{3}$ and $r^{*} \geq 0$, define, for each $\left(x, u_{c}, w_{c}\right) \in \mathbb{R}^{n} \times$ $\mathbb{R}^{m_{c}} \times \mathbb{R}^{d_{c}}$ and $r \geq \bar{r}^{*}$, the function

$$
\Gamma_{c}\left(x, u_{c}, r\right):=\left\{\begin{array}{rr}
\sup _{w_{c} \in \Psi_{c}^{w}(x)} \sup _{\xi \in F\left(x, u_{c}, w_{c}\right)}\langle\nabla V(x), \xi\rangle \\
\quad+\frac{1}{2} \alpha_{3}\left(|x|_{\mathcal{A}}\right) \\
-\infty & \text { if }\left(x, u_{c}\right) \in \Delta_{c}(r, C), \\
\text { otherwise }
\end{array}\right.
$$


and, for each $\left(x, u_{d}, w_{d}\right) \in \mathbb{R}^{n} \times \mathbb{R}^{m_{d}} \times \mathbb{R}^{d_{d}}$ and $r \geq r^{*}$, the function

$\Gamma_{d}\left(x, u_{d}, r\right):=\left\{\begin{array}{rr}\sup _{w_{d} \in \Psi_{d}^{w}(x)} \sup _{\xi \in G\left(x, u_{d}, w_{d}\right)} V(\xi)-V(x) \\ \\ -\infty & +\frac{1}{2} \alpha_{3}\left(|x|_{\mathcal{A}}\right) \\ \text { if }\left(x, u_{d}\right) \in \Delta_{d}(r, D), & \text { otherwise. }\end{array}\right.$

When these functions and the system satisfy further properties introduced below, the existence of a $w$-robustly stabilizing feedback law is guaranteed.

TheOREM 4.1. Given a compact set $\mathcal{A} \subset \mathbb{R}^{n}$ and a hybrid system $\mathcal{H}=(C, F, D, G)$ satisfying the hybrid basic conditions, suppose there exists a robust control Lyapunov function $V$ with $\mathcal{U}$ controls for $\mathcal{H}_{u, w}$ that is continuously differentiable on a neighborhood of $\Pi_{c}(C) \cap \mathcal{I}\left(r^{*}\right)$, where $r^{*}$ comes from Definition 3.6 . Furthermore, suppose the following conditions hold:

R1) The set-valued maps $\Psi_{c}^{u}$ and $\Psi_{d}^{u}$ are lower semicontinuou $\S^{4}$ with convex values.

R2) For every $r>r^{*}$ and for every $x \in \Pi_{c}(C) \cap \mathcal{I}(r)$, the function $u_{c} \mapsto \Gamma_{c}\left(x, u_{c}, r\right)$ is convex on $\Psi_{c}^{u}(x)$ and, for every $r>r^{*}$ and every $x \in \Pi_{d}(D) \cap \mathcal{I}(r)$, the function $u_{d} \mapsto \Gamma_{d}\left(x, u_{d}, r\right)$ is convex on $\Psi_{d}^{u}(x)$.

R3) The set $\mathcal{W}$ is closed and the set-valued maps $\Psi_{c}^{w}$ and $\Psi_{d}^{w}$ are outer semicontinuous, locally bounded, and nonempty for each $x \in \Pi_{c}(C) \cap \mathcal{I}\left(r^{*}\right)$ and each $x \in \Pi_{d}(D) \cap$ $\mathcal{I}\left(r^{*}\right)$, respectively.

Then, for each $r>r^{*}$, the set $\mathcal{A}$ is w-robustly uniformly globally asymptotically stabilizable with residual

$$
\widetilde{\mathcal{A}}=\left\{x \in \mathbb{R}^{n}: V(x) \leq r\right\}
$$

for $\mathcal{H}_{u, w}^{\mathcal{I}}$ by a state-feedback law $\left(\kappa_{c}, \kappa_{d}\right)$ that is continuous on $\left(\Pi_{c}(C) \cap \mathcal{I}(r)\right) \times\left(\Pi_{d}(D) \cap \mathcal{I}(r)\right)$, where $\mathcal{H}_{u, w}^{\mathcal{I}}$ is the restriction of $\mathcal{H}_{u, w}$ to $\mathcal{I}(r)$ given by

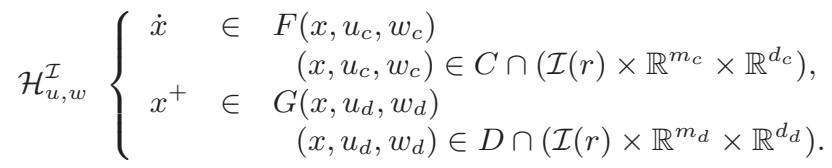

In particular, for each $r>r^{*}$, there exists a state-feedback law $\left(\kappa_{c}, \kappa_{d}\right)$ with $\kappa_{c}$ continuous on $\Pi_{c}(C) \cap \mathcal{I}(r)$ and $\kappa_{d}$ continuous on $\Pi_{d}(D) \cap \mathcal{I}(r)$ defining an admissible control $u=\left(\kappa_{c}, \kappa_{d}\right)$ that renders the compact set $\widetilde{\mathcal{A}}$ in (11) $w$ robustly uniformly globally asymptotically stable relative to $\mathcal{A}$ for $\mathcal{H}_{u, w}^{\mathcal{I}}$.

EXAMPLE 4.2. (controlled pendulum with impacts (revisited)) A robust control Lyapunov function satisfying the conditions in Theorem 4.1 was constructed in Example 3.7.

\footnotetext{
${ }^{4}$ A set-valued map $S: \quad \mathbb{R}^{n} \rightrightarrows \mathbb{R}^{m}$ is lower semicontinuous if for each $x \in \mathbb{R}^{n}$ one has that $\liminf _{x_{i} \rightarrow x} S\left(x_{i}\right) \supset S(x)$, where $\liminf _{x_{i} \rightarrow x} S\left(x_{i}\right)=$ $\left\{z: \forall x_{i} \rightarrow x, \exists z_{i} \rightarrow z\right.$ s.t. $\left.z_{i} \in S\left(x_{i}\right)\right\}$ is the inner limit of $S$ (see [10, Chapter 5.B]). By lower semicontinuity of a setvalued map $S$ with not open $\operatorname{dom} S$ we mean that the trivial extension of $S$ proposed in [11, Lemma 4.2] is lower semicontinuous.
}

Conditions R1) and R3) immediately hold from the constructions therein. The definition of $\Gamma_{c}$ above gives, for each $r \geq 0$,

$\Gamma_{c}\left(x, u_{c}, r\right)=\left\{\begin{array}{c}\sup _{w_{c} \in \Psi_{c}^{w}(x)}\left[4 x_{1} x_{2}+2 x_{2}^{2}+2\left(-a \sin x_{1}\right.\right. \\ \left.-\left(b+w_{c, 2}\right) x_{2}+u_{c, 1}+w_{c, 1}\right)\left(x_{2}+x_{1}\right) \\ \left.\quad+\alpha_{3}\left(|x|_{\mathcal{A}}\right)\right] \\ \text { if }\left(x, u_{c}\right) \in \Delta_{c}(r, C), \\ -\infty \\ \text { otherwise }\end{array}\right.$

while the definition of $\Gamma_{d}$ above gives, for each $r \geq 0$,

$$
\Gamma_{d}\left(x, u_{d}, r\right)=\left\{\begin{array}{r}
\sup _{w_{d} \in \Psi_{d}^{w}(x)}\left[-2 x_{1}^{2}\left(1-\left(1+\widetilde{\rho}\left(u_{d}\right)\right)^{2}\right)\right. \\
-x_{2}^{2}\left(1-\left(e\left(u_{d}\right)+w_{d}\right)^{2}\right) \\
-2 x_{1} x_{2}\left(1+\left(1+\widetilde{\rho}\left(u_{d}\right)\right)\left(e\left(u_{d}\right)+w_{d}\right)\right) \\
\left.+\alpha_{3}\left(|x|_{\mathcal{A}}\right)\right] \\
\text { if }\left(x, u_{d}\right) \in \Delta_{d}(r, D), \\
-\infty
\end{array}\right.
$$

Then, R2) holds. Hence, since $r^{*}=0$, according to Theorem 4.1. the hybrid system in Example 2.1 has its origin $w$-robustly practically uniformly globally asymptotically stabilizable. We will see in Example 5.4 that a non-practical property already holds and that a stabilizing state-feedback law can actually be synthesized.

The result above guarantees a robust stabilizability property that either has a residual or is practical. For robust stabilizability of a compact set, extra conditions are required to hold nearby the compact set. For continuous-time systems, such conditions correspond to the so-called small control property [14, 6, 8]. To that end, given a compact set $\mathcal{A}$ and a robust control Lyapunov function $V$ as in Definition 3.6] define, for each $(x, r) \in \mathbb{R}^{n} \times \mathbb{R}_{\geq 0}$, the set-valued man

$$
\begin{aligned}
& \widehat{S}_{c}(x, r):= \begin{cases}S_{c}(x, r) & \text { if } r>0 \\
\kappa_{c, 0}(x) & \text { if } r=0 \\
S_{d}(x, r) & \text { if } r>0 \\
\kappa_{d, 0}(x) & \text { if } r=0\end{cases}
\end{aligned}
$$

where $\kappa_{c, 0}: \mathbb{R}^{n} \rightarrow \mathcal{U}_{c}$ and $\kappa_{d, 0}: \mathbb{R}^{n} \rightarrow \mathcal{U}_{d}$ induce forward invariance of $\mathcal{A}$ for $\mathcal{H}_{u, w}$, that is,

R4) Every maximal solution $\left(\phi, w_{c}\right)$ to

$$
\dot{x} \in F\left(x, \kappa_{c, 0}(x), w_{c}\right) \quad\left(x, \kappa_{c, 0}(x), w_{c}\right) \in C
$$

from $\mathcal{A}$ is such that the $\phi$ component satisfies $|\phi(t, 0)|_{\mathcal{A}}=$ 0 for all $(t, 0) \in \operatorname{dom}\left(\phi, w_{c}\right)$.

R5) Every maximal solution $\left(\phi, w_{d}\right)$ to

$$
x^{+} \in G\left(x, \kappa_{d, 0}(x), w_{d}\right) \quad\left(x, \kappa_{d, 0}(x), w_{d}\right) \in D
$$

from $\mathcal{A}$ is such that the $\phi$ component satisfies $|\phi(0, j)|_{\mathcal{A}}=$ 0 for all $(0, j) \in \operatorname{dom}\left(\phi, w_{d}\right)$.

\footnotetext{
${ }^{5}$ Note that if either $\Pi_{c}(C)$ or $\Pi_{d}(D)$ do not intersect the compact set $\mathcal{A}$, then neither the existence of the functions $\kappa_{c, 0}$ or $\kappa_{d, 0}$, respectively, nor lower semicontinuity at $r=0$ are needed, since R4) and R5) would hold for free.
} 
Under the conditions in Theorem 4.1 with $r^{*}=0$, the maps in (12) are lower semicontinuous for every $r>0$. To be able to make continuous selections at $\mathcal{A}$, these maps are further required to be lower semicontinuous for $r=0$. These conditions resemble those already reported in [6] for continuoustime systems.

THEOREM 4.3. Under the conditions of Theorem 4.1 and when $r^{*}=0$, if there exist continuous functions $\kappa_{c, 0}: \mathbb{R}^{n} \rightarrow$ $\mathcal{U}_{c}$ and $\kappa_{d, 0}: \mathbb{R}^{n} \rightarrow \mathcal{U}_{d}$ such that conditions $\left.R_{4}\right)$ and $R 5$ ) hold, and

R6) The set-valued map $\widehat{S}_{c}$ is lower semicontinuous at each $x \in \Pi_{c}(C) \cap \mathcal{I}(0)$;

R7) The set-valued map $\widehat{S}_{d}$ is lower semicontinuous at each $x \in \Pi_{d}(D) \cap \mathcal{I}(0)$;

R8) The hybrid exosystem $\mathcal{H}_{e}$ in (2) satisfies the hybrid basic conditions;

then $\mathcal{A}$ is w-robustly uniformly globally asymptotically stabilizable for $\mathcal{H}_{u, w}$ by a continuous state-feedback pair $\left(\kappa_{c}, \kappa_{d}\right)$.

\section{CONSTRUCTIVE DESIGN OF ROBUSTLY STABILIZING FEEDBACK LAWS}

We show that, under further conditions, the results in Section 4 lead to a constructive design procedure of statefeedback control laws that induce $w$-robust asymptotic stability. The key idea is to define a selection from the "regulation map" that can be synthesized (or computed) for given system data and RCLF.

Recalling the construction of $\Gamma_{c}$ and $\Gamma_{d}$ in Section 4 we evaluate these functions at points $\left(x, u_{c}, r\right)$ and $\left(x, u_{d}, r\right)$ with $r=V(x)$ to define the functions

$$
\begin{aligned}
\left(x, u_{c}\right) & \mapsto \Upsilon_{c}\left(x, u_{c}\right):=\Gamma_{c}\left(x, u_{c}, V(x)\right), \\
\left(x, u_{d}\right) & \mapsto \Upsilon_{d}\left(x, u_{d}\right):=\Gamma_{d}\left(x, u_{d}, V(x)\right)
\end{aligned}
$$

and the set-valued maps

$$
\left.\begin{array}{l}
\mathcal{T}_{c}(x):=\Psi_{c}^{u}(x) \cap\left\{u_{c} \in \mathcal{U}_{c}: \Upsilon_{c}\left(x, u_{c}\right) \leq 0\right\}, \\
\mathcal{T}_{d}(x):=\Psi_{d}^{u}(x) \cap\left\{u_{d} \in \mathcal{U}_{d}: \Upsilon_{d}\left(x, u_{d}\right) \leq 0\right.
\end{array}\right\} .
$$

Furthermore, define

$$
R_{c}:=\Pi_{c}(C) \cap\left\{x \in \mathbb{R}^{n}: V(x)>0\right\}
$$

and

$$
R_{d}:=\Pi_{d}(D) \cap\left\{x \in \mathbb{R}^{n}: V(x)>0\right\} .
$$

When, for each $x$, the functions $u_{c} \mapsto \Upsilon_{c}\left(x, u_{c}\right)$ and $u_{d} \mapsto$ $\Upsilon_{d}\left(x, u_{c}\right)$ are convex, and the set-valued maps $\Psi_{c}^{u}$ and $\Psi_{d}^{u}$ have nonempty closed convex values on $R_{c}$ and $R_{d}$, respectively, we have that $\mathcal{T}_{c}$ and $\mathcal{T}_{d}$ have nonempty convex closed values on (15) and on (16), respectively; this property follows from [5, Proposition 4.4]. Then, $\mathcal{T}_{c}$ and $\mathcal{T}_{d}$ have unique elements of minimum norm on $R_{c}$ and $R_{d}$, respectively, and their minimal selections

$$
\rho_{c}: R_{c} \rightarrow \mathcal{U}_{c}, \quad \rho_{d}: R_{d} \rightarrow \mathcal{U}_{d}
$$

are given by

$$
\begin{array}{ll}
\rho_{c}(x):=\arg \min \left\{\left|u_{c}\right|:\right. & \left.u_{c} \in \mathcal{T}_{c}(x)\right\} \\
\rho_{d}(x):=\arg \min \left\{\left|u_{d}\right|:\right. & \left.u_{d} \in \mathcal{T}_{d}(x)\right\}
\end{array}
$$

Moreover, as the following result states, these selections are continuous under further properties of $\Psi_{c}^{u}$ and $\Psi_{d}^{u}$.

TheOREM 5.1. Given a compact set $\mathcal{A} \subset \mathbb{R}^{n}$ and a hybrid system $\mathcal{H}_{u, w}=(C, F, D, G)$ satisfying the hybrid basic conditions, suppose there exists a robust control Lyapunov function $V$ with $\mathcal{U}$ controls for $\mathcal{H}_{u, w}$ that is continuously differentiable on a neighborhood of $\Pi_{c}(C) \cap \mathcal{I}\left(r^{*}\right)$, where $r^{*}$ comes from Definition 3.6. Furthermore, suppose conditions R1)-R3) in Theorem 4.1 hold. Then, for each $r>r^{*}$, the state-feedback law pair

$$
\rho_{c}: R_{c} \cap \mathcal{I}(r) \rightarrow \mathcal{U}_{c}, \quad \rho_{d}: R_{d} \cap \mathcal{I}(r) \rightarrow \mathcal{U}_{d}
$$

defined as

$$
\begin{aligned}
& \rho_{c}(x):=\arg \min \left\{\left|u_{c}\right|: u_{c} \in \mathcal{T}_{c}(x)\right\} \\
& \forall x \in R_{c} \cap \mathcal{I}(r) \\
& \rho_{d}(x):=\arg \min \left\{\left|u_{d}\right|: u_{d} \in \mathcal{T}_{d}(x)\right\} \\
& \forall x \in R_{d} \cap \mathcal{I}(r)
\end{aligned}
$$

renders the compact set

$$
\widetilde{\mathcal{A}}=\left\{x \in \mathbb{R}^{n}: V(x) \leq r\right\}
$$

w-robustly uniformly globally asymptotically stable for $\mathcal{H}_{u, w}^{\mathcal{I}}$ relative to $\mathcal{A}$, where $\mathcal{H}_{u, w}^{\mathcal{I}}$ is the restriction of $\mathcal{H}_{u, w}$ to $\mathcal{I}(r)$ given as in Theorem 4.1 .

Furthemore, if the set-valued maps $\Psi_{c}^{u}$ and $\Psi_{d}^{u}$ have closed graph then $\rho_{c}$ and $\rho_{d}$ are continuous.

REMARK 5.2. When bounds (8) and (9) hold for functions $\alpha_{3, c}$ and $\alpha_{3, d}$, respectively, the expressions of the pointwise minimum norm control laws (17) and (18) can be rewritten in terms of those functions (instead of a common function $\alpha_{3}$ ) by defining, respectively, $\mathcal{T}_{c}$ and $\mathcal{T}_{d}$ using $\alpha_{3, c}$ and $\alpha_{3, d}$ in place of $\alpha_{3}$.

The state-feedback law (19)-(20) asymptotically stabilizes $\widetilde{\mathcal{A}}$ for $\mathcal{H}_{u, w}^{\mathcal{I}}$, but not necessarily for $\mathcal{H}_{u, w}$, as without an appropriate extension of these laws to $\Pi_{c}(C)$ and $\Pi_{d}(D)$, respectively, there could exist solutions to the closed-loop system that jump out of $\widetilde{\mathcal{A}}$. This point motivates the (nonpractical, and stronger) result that we present next.

Following the ideas behind Theorem 4.3 we extend the pointwise minimum norm state-feedback control law in Theorem 5.1 so as to $w$-robustly globally asymptotically stabilize a compact set $\mathcal{A}$. To that end, given a compact set $\mathcal{A}$ and a robust control Lyapunov function $V$ satisfying Definition 3.6 for each $x \in \mathbb{R}^{n}$, define

$$
\begin{aligned}
& \mathcal{T}_{c}^{\prime}(x):=\Psi_{c}^{u}(x) \cap S_{c}^{\prime}(x, V(x)), \\
& \mathcal{T}_{d}^{\prime}(x):=\Psi_{d}^{u}(x) \cap S_{d}^{\prime}(x, V(x)),
\end{aligned}
$$

where, for each $x \in \mathbb{R}^{n}$ and each $r \geq 0$,

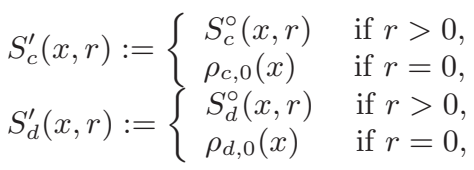


$S_{c}^{\circ}(x, r)=\left\{\begin{array}{rr}\left\{u_{c} \in \mathcal{U}_{c}: \Gamma_{c}\left(x, u_{c}, r\right) \leq 0\right\} & \text { if } x \in \Pi_{c}(C) \cap \mathcal{I}(r), \\ \mathbb{R}^{m_{c}} & \text { otherwise, }\end{array}\right.$
$S_{d}^{\circ}(x, r)= \begin{cases}\left\{u_{d} \in \mathcal{U}_{d}:\right. & \left.\Gamma_{d}\left(x, u_{d}, r\right) \leq 0\right\} \\ & \text { if } x \in \Pi_{d}(D) \cap \mathcal{I}(r), \\ \mathbb{R}^{m_{d}} & \text { otherwise, }\end{cases}$

and the feedback law pair

$$
\begin{aligned}
& \rho_{c, 0}: \mathbb{R}^{n} \rightarrow \mathcal{U}_{c} \\
& \rho_{d, 0}: \mathbb{R}^{n} \rightarrow \mathcal{U}_{d}
\end{aligned}
$$

induces (strong) forward invariance of $\mathcal{A}$ as stated in R4) (with $\kappa_{c, 0}=\rho_{c, 0}$ ) and R5) (with $\kappa_{d, 0}=\rho_{d, 0}$ ) in Section 4 Note that under the conditions in Theorem 5.1 the maps in (12) are lower semicontinuous for every $r>0$. To be able to make continuous selections at $\mathcal{A}$, these maps are further required to be lower semicontinuous for $r=0$.

TheOREM 5.3. Under the conditions of Theorem 5.1 and when $r^{*}=0$, if there exists a feedback law pair $\left(\rho_{c, 0}: \mathbb{R}^{n} \rightarrow\right.$ $\left.\mathcal{U}_{c}, \rho_{d, 0}: \mathbb{R}^{n} \rightarrow \mathcal{U}_{d}\right)$ such that $R_{4}$ ) and R5) in Section 4 hold, and

M1) The set-valued map $\mathcal{T}_{c}^{\prime}$ in (21) is lower semicontinuous at each $x \in \Pi_{c}(C) \cap \mathcal{I}(0)$;

M2) The set-valued map $\mathcal{T}_{d}^{\prime}$ in (22) is lower semicontinuous at each $x \in \Pi_{d}(D) \cap \mathcal{I}(0)$;

hold, then the state-feedback law pair

$$
\rho_{c}: \Pi_{c}(C) \rightarrow \mathcal{U}_{c}, \quad \rho_{d}: \Pi_{d}(D) \rightarrow \mathcal{U}_{d}
$$

defined as

$$
\begin{array}{cc}
\rho_{c}(x):=\arg \min \left\{\left|u_{c}\right|: u_{c} \in \mathcal{T}_{c}^{\prime}(x)\right\} & \forall x \in \Pi_{c}(C) \\
\rho_{d}(x):=\arg \min \left\{\left|u_{d}\right|: u_{d} \in \mathcal{T}_{d}^{\prime}(x)\right\} & \forall x \in \Pi_{d}(D)
\end{array}
$$

renders the compact set $\mathcal{A}$ w-robustly uniformly globally asymptotically stable for $\mathcal{H}_{u, w}$. Furthermore, if the set-valued maps $\Psi_{c}$ and $\Psi_{d}$ have closed graph and $\left(\rho_{c, 0}, \rho_{d, 0}\right)(\mathcal{A})=0$, then $\rho_{c}$ and $\rho_{d}$ are continuous.

We revisit our running example and synthesize a stabilizing feedback. Simulations validate the results.

EXAMPLE 5.4. (controlled pendulum with impacts (revisited)) From the constructions of $\Gamma_{c}$ and $\Gamma_{d}$ in Example 4.2. the set-valued map $\mathcal{T}_{c}$ is given by

$$
\begin{aligned}
\left\{u_{c} \in \mathbb{R}\right. & \times\left[-\frac{\pi}{2}, \min \left\{x_{1}, 0\right\}\right]: 4 x_{1} x_{2}+2 x_{2}^{2} \\
& +2\left(-a \sin x_{1}-b x_{2}+u_{c, 1}\right)\left(x_{2}+x_{1}\right)+\lambda x^{\top} x \\
& \left.+2\left|x_{2}+x_{1}\right|\left(\bar{w}_{c, 2}\left|x_{2}\right|+\bar{w}_{c, 1}\right) \leq 0\right\}
\end{aligned}
$$

for each $x \in \Pi_{c}(C) \cap\left\{x \in \mathbb{R}^{2}: V(x)>0\right\}$.

Proceeding in the same way, the set-valued map $\mathcal{T}_{d}$ is given by

$$
\begin{aligned}
\left\{u_{d}\right. & \in\left[x_{1}, 0\right]:-2 x_{1}^{2}\left(1-\left(1+\widetilde{\rho}\left(u_{d}\right)\right)^{2}\right) \\
& \left.-x_{2}^{2}\left(1-\left(e\left(u_{d}\right)+e_{1}-e_{0}\right)^{2}\right)+\lambda x^{\top} x \leq 0\right\}
\end{aligned}
$$

${ }^{6}$ With $\kappa_{c, 0}=\rho_{c, 0}$ and $\kappa_{d, 0}=\rho_{d, 0}$. for each $x \in \Pi_{d}(D) \cap\left\{x \in \mathbb{R}^{2}: V(x)>0\right\}$, where we dropped the term $-2 x_{1} x_{2}\left(1+\left(1+\widetilde{\rho}\left(u_{d}\right)\right)\left(e\left(u_{d}\right)+w_{d}\right)\right)$ since on $D$ we have that $x_{1} x_{2} \geq 0$.

Now, we synthesize the control law using Theorem 5.3 . Defining $\psi_{0}(x):=4 x_{1} x_{2}+2 x_{2}^{2}+2\left(-a \sin x_{1}-b x_{2}\right)\left(x_{2}+x_{1}\right)+$ $\lambda x^{\top} x, \psi_{0}^{w}(x):=2\left|x_{2}+x_{1}\right|\left(\bar{w}_{c, 2}\left|x_{2}\right|+\bar{w}_{c, 1}\right)$, and $\psi_{1}(x):=$ $2\left(x_{1}+x_{2}\right)$, the map in (26) can be rewritten as

$$
\begin{aligned}
\mathcal{T}_{c}(x)=\left\{u_{c}\right. & \in \mathbb{R} \times\left[-\frac{\pi}{2}, \min \left\{x_{1}, 0\right\}\right]: \\
& \left.\psi_{0}(x)+\psi_{0}^{w}(x)+\psi_{1}(x) u_{c, 1} \leq 0\right\}
\end{aligned}
$$

for each $x \in \Pi_{c}(C) \cap\left\{x \in \mathbb{R}^{2}: V(x)>0\right\}$. To determine the pointwise minimum norm control selection according to (17), note that, when $\psi_{0}(x)+\psi_{0}^{w}(x) \leq 0$, the pointwise minimum norm control selection is $u_{c, 1}=0$ and that, when $\psi_{0}(x)+\psi_{0}^{w}(x)>0$, is given by

$$
-\frac{\left(\psi_{0}(x)+\psi_{0}^{w}(x)\right) \psi_{1}(x)}{\psi_{1}^{2}(x)}=-\frac{\psi_{0}(x)+\psi_{0}^{w}(x)}{\psi_{1}(x)}
$$

which leads to $\psi_{0}(x)+\psi_{0}^{w}(x)+\psi_{1}(x) u_{c, 1}=0$. Then, the pointwise minimum norm control selection is given by

$$
\begin{aligned}
\rho_{c, 1}(x) & := \begin{cases}-\frac{\psi_{0}(x)+\psi_{0}^{w}(x)}{\psi_{1}(x)} & \psi_{0}(x)+\psi_{0}^{w}(x)>0 \\
0 & \psi_{0}(x)+\psi_{0}^{w}(x) \leq 0\end{cases} \\
\rho_{c, 2}(x) & :=0
\end{aligned}
$$

on $\Pi_{c}(C) \cap\left\{x \in \mathbb{R}^{2}: V(x)>0\right\}$. Note that there is no division by zero in the construction of $\rho_{c, 1}$ since, when $\psi_{1}(x)=$ 0 we have that $\mathcal{T}_{c}(x)$ implies that $\psi_{0}(x)+\psi_{0}^{w}(x) \leq 0$, in which case, $\rho_{c, 1}$ is defined as zero.

Next, we design the state-feedback law to be used at jumps. According to (18), since $\widetilde{\rho}$ maps to $(-1,0)$, e to $\left(e_{0}, e_{1}\right)$, and $w_{d} \in\left[0, e_{1}-e_{0}\right]$, the pointwise minimum norm control selection is given by

$$
\rho_{d}(x):=0 .
$$

for each $x \in \Pi_{d}(D) \cap\left\{x \in \mathbb{R}^{2}: V(x)>0\right\}$. Since $\rho_{c, 2}=$ $\rho_{d}$, the selection above uniquely defines the input $\mu$.

Figures 1 4 show closed-loop trajectories using the designed pointwise minimum norm control law $\left(\left(\rho_{c, 1}, \rho_{c, 2}\right), \rho_{d}\right)$. The restitution function used is linear with $e_{0}=\frac{1}{3}$ and $e_{1}=\frac{2}{3}$, and the function $\widetilde{\rho}$ is constant and equal to $-\frac{1}{20}$. The simulation results show convergence to the set $\mathcal{A}=\{(0,0)\}$, even under the presence of perturbations. For simplicity, the simulations are performed under constant disturbances $\left(w_{c}, w_{d}\right)$, for different values of $w_{c}$ and $w_{d}$.

The plots in Figure 1 and Figure 2 correspond to solutions for different values of $w_{c}$ and with $w_{d}=0$. The velocity component jumps at the impact time and then rapidly gets close to nearby zero. The larger the disturbance, the longer it takes for the solutions to converge. While not being part of the design procedure, the control law $\rho_{c}$ steers the solutions to the origin from within the flow set. In fact, as the solutions approach a neighborhood of $\mathcal{A}$, they evolve nearby the manifold $x_{1}+x_{2}=0$, which leads to large input values.

The plots in Figure 3 and Figure 4 correspond to solutions for different values of $w_{d}$ and with $w_{c}=0$. Since the disturbance $w_{d}$ is positive and captures the uncertainty in the restitution coefficient function, large values of the disturbance cause large peaks after every jump as well as more jumps during the transient, when compared to the results in

${ }^{7}$ See [6, Chapter 4]. 
Figure 1] and Figure 2, After a few jumps, the solutions approach a neighborhood of $\mathcal{A}$ along the manifold $x_{1}+x_{2}=0$.
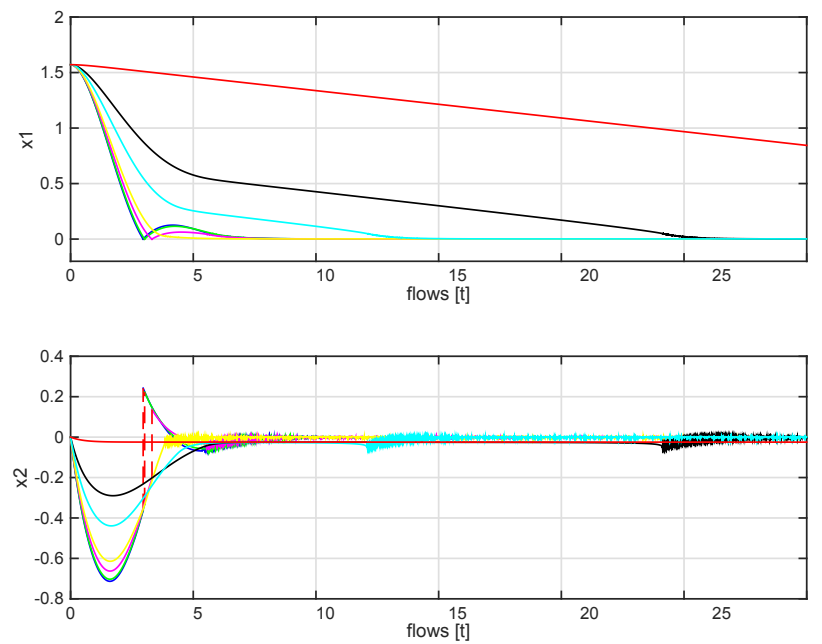

Figure 1: Closed-loop trajectories as a function of flow time $t$ to the system in Example 5.4 starting from $\phi(0,0)=$ $(1.5707,0)$ (marked with $\star)$. The disturbances used are constant and with the following values: for each $i \in\{1,2\}$, $w_{c, i}=0$ (blue), $w_{c, i}=0.01$ (green), $w_{c, i}=0.05$ (magenta), $w_{c, i}=0.1$ (yellow), $w_{c, i}=0.3$ (cyan), $w_{c, i}=0.5$ (black), $w_{c, i}=1$ (red); $w_{d}=0$ (all simulations).

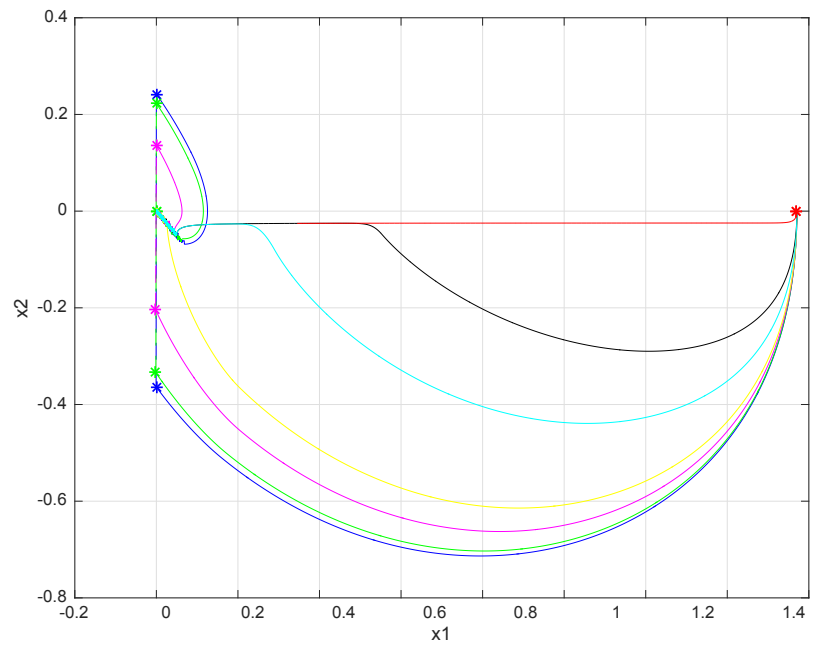

Figure 2: Closed-loop trajectories on the plane to the system in Example 5.4 starting from $\phi(0,0)=(1.5707,0)$ (marked with $\star$ ). The disturbances used are constant and with the following values: for each $i \in\{1,2\}, w_{c, i}=0$ (blue), $w_{c, i}=0.01$ (green), $w_{c, i}=0.05$ (magenta), $w_{c, i}=0.1$ (yellow), $w_{c, i}=0.3$ (cyan), $w_{c, i}=0.5$ (black), $w_{c, i}=1$ (red); $w_{d}=0$ (all simulations). The $\star$ 's after the initial interval of flow in the plot of the solutions denote the values of the solution before and after the jump.
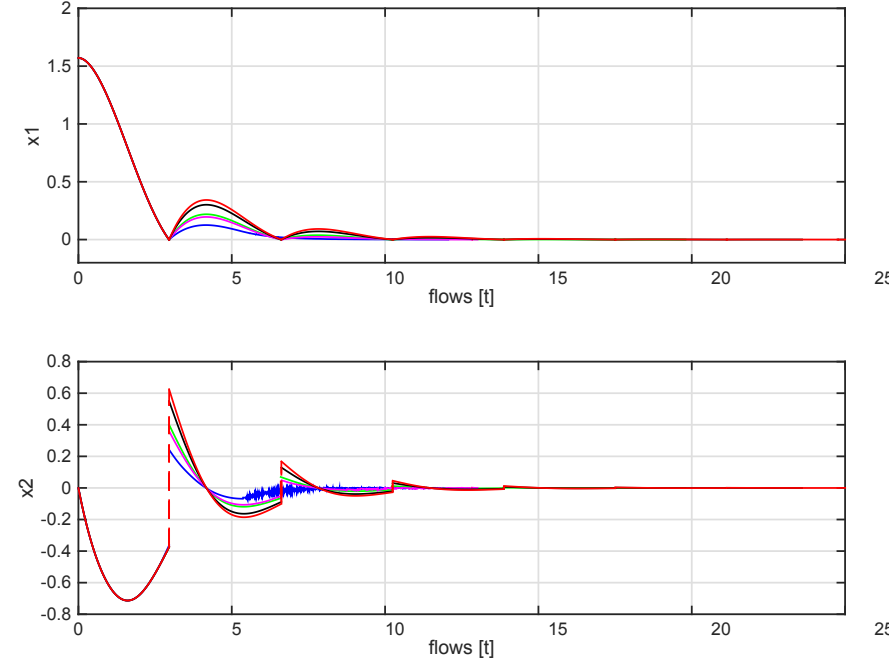

Figure 3: Closed-loop trajectories as a function of flow time $t$ to the system in Example 5.4 starting from $\phi(0,0)=$ $(1.5707,0)$ (marked with $\star$ ). The disturbances used are constant and with the following values: for each $i \in\{1,2\}$, $w_{c, i}=0$ (all simulations); $w_{d}=0 ;$ (blue), $w_{d}=0.3$ (magenta), $w_{d}=0.4$ (green), $w_{d}=0.8$ (black), $w_{d}=1$ (red).

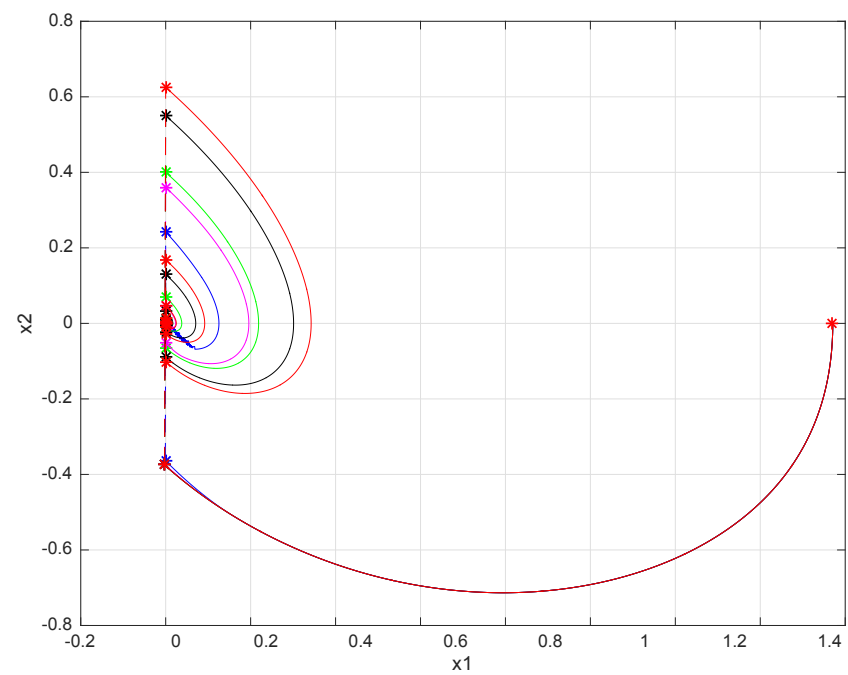

Figure 4: Closed-loop trajectories on the plane to the system in Example 5.4 starting from $\phi(0,0)=(1.5707,0)$ (marked with $\star$ ). The disturbances used are constant and with the following values: for each $i \in\{1,2\}, w_{c, i}=0$ (all simulations); $w_{d}=0$; (blue), $w_{d}=0.3$ (magenta), $w_{d}=0.4$ (green), $w_{d}=0.8$ (black), $w_{d}=1$ (red). The $\star$ 's after the initial interval of flow in the plot of the solutions denote the values of the solution before and after the jump. 


\section{CONCLUSION}

For a wide class of hybrid systems given in terms of hybrid inclusions with inputs and disturbances, we presented CLF-based results to guarantee the existence of stabilizing state-feedback controllers and to constructively design them. When a CLF is available and the required conditions hold, a state-feedback law with pointwise minimum norm can be constructed to asymptotically stabilize a compact set with robustness to disturbances. A remarkable feature of this controller construction is that it guarantees $w$-robust asymptotic stability of the closed-loop system for any admissible disturbance taking values from (the $w$ components of) points in the flow set or jump set. Such disturbances can indeed be large, unlike the disturbances allowed in our previous nominal robustness results in 7 , and, as a difference to input-to-state stability-based results (see [1]), at times can be fully rejected.

The implementation of the proposed feedback laws requires careful treatment to allow for computation in realistic systems. In particular, the computations involved in determining the minimizers in the state-feedback laws (17) and (18) require a nonzero amount of time to terminate. A sample-and-hold or event-triggered implementation of such laws would require variables that trigger the computation events, allow the computations to terminate, and upon termination of the computations, update the inputs to the hybrid system under control. Recent results suggest that, as long as the time for the computations to terminate can be made sufficiently small, it is possible to implement such laws while preserving the stability properties semiglobally and practically 12. Handling the challenges in performing such computations is part of current research efforts.

Finally, the proposed state-feedback law with pointwise minimum norm is expected to also induce an optimality property of the closed-loop system. Using inverse optimality ideas, the robust stabilization problem solved in this paper can be recast as a two-player zero-sum hybrid dynamical game. Under appropriate assumptions, we conjecture that the proposed control law suboptimally solves such hybrid game with a meaningful cost function.

\section{REFERENCES}

[1] C. Cai and A. R. Teel. Characterizations of input-to-state stability for hybrid systems. Syst. \&6 Cont. Letters, 58:47-53, 2009.

[2] F. Clarke. Optimization and Nonsmooth Analysis.
SIAM's Classic in Applied Mathematics, Philadelphia, 1990.

[3] F. Clarke, Y. S. Ledyaev, L. Rifford, and R. Stern. Feedback stabilization and Lyapunov functions. SIAM: Journal of Control and Optimization, 39(1):25-48, 2000.

[4] S. Di Cairano, W. M. H. Heemels, M. Lazar, and A. Bemporad. Stabilizing dynamic controllers for hybrid systems: a hybrid control Lyapunov function approach. IEEE Transactions on Automatic Control, 59(10):2629-2643, 2014.

[5] R. Freeman and P. Kokotovic. Inverse optimality in robust stabilization. SIAM Journal of Control and Optimization, 34:1365-1391, 1996.

[6] R. A. Freeman and P. V. Kokotovic. Robust Nonlinear Control Design: State-Space and Lyapunov Techniques. Birkhauser, 1996.

[7] R. Goebel, R. G. Sanfelice, and A. R. Teel. Hybrid Dynamical Systems: Modeling, Stability, and Robustness. Princeton University Press, New Jersey, 2012.

[8] M. Krstic and H. Deng. Stabilization of nonlinear uncertain systems. Springer-Verlag New York, 1998.

[9] M. Robles and R. G. Sanfelice. Hybrid controllers for tracking of impulsive reference trajectories: A hybrid exosystem approach. In Proc. 14th International Conference Hybrid Systems: Control and Computation, pages 231-240, 2011.

[10] R. Rockafellar and R. J.-B. Wets. Variational Analysis. Springer, Berlin Heidelberg, 1998.

[11] R. G. Sanfelice. On the existence of control Lyapunov functions and state-feedback laws for hybrid systems. IEEE Transactions on Automatic Control, 58(12):3242-3248, December 2013.

[12] R. G. Sanfelice. Computationally tractable implementations of pointwise minimum norm state-feedback laws for hybrid systems. In Proceedings of the American Control Conference (to appear), 2016.

[13] E. Sontag and H. Sussmann. General classes of control-Lyapunov functions. In Stability theory: Hurwitz Centenary Conference, Centro Stefano Franscini, Ascona, 1995. 1996.

[14] E. D. Sontag. A 'universal' construction of Artstein's theorem on nonlinear stabilization. Systems and Control Letters, 13:117-123, 1989. 